\title{
Acute kidney injury in parturients with severe preeclampsia
}

\author{
Sun-Kyung Park ${ }^{1} \cdot$ Min Hur $^{1} \cdot$ Won Ho Kim ${ }^{1}$
}

Received: 14 June 2018 / Accepted: 18 July 2018 / Published online: 24 July 2018

(c) Japanese Society of Anesthesiologists 2018

Keywords Acute kidney injury · Cesarean section · Preeclampsia

To the Editor:

We read with interest the study by Mazda et al. [1] regarding postoperative acute kidney injury (AKI) in parturients with severe preeclamsia. We would like to discuss the clinical implication of the study. First, the effect of hydroxyl ethyl starch (HES) administration was not evaluated fully. Clinical outcomes other than AKI were not compared with a control group. Although not clearly shown, the number of patients with proteinuria or elevated serum creatinine seems to be very small. The incidence of AKI and the impact of HES on AKI may be different in the more selected patients with severe preeclampsia with renal dysfunction [2]. Second, the serum creatinine measurement may not be a reliable estimation of glomerular filtration rate (GFR) in these parturients [3]. Delivery itself and different oral intakes after surgery may influence serum creatinine and following creatinine after delivery may not be accurate. GFR increases up to 50\% during pregnancy and continue at levels $20 \%$ above normal at postpartum week [4]. Diagnosis of AKI in these patients using other measurement of GFR or more sensitive biomarkers may be required.

\section{Compliance with ethical standards}

Conflict of interest No competing interest declared.

\section{References}

1. Mazda Y, Tanaka M, Terui K, Nagashima S, Inoue R. Postoperative renal function in parturients with severe preeclampsia who underwent cesarean delivery: a retrospective observational study. J Anesth. 2018;32:447-51.

2. Awowole IO, Omitinde OS, Arogundade FA, Bola-Oyebamiji SB, Adeniyi OA. Pregnancy-related acute kidney injury requiring dialysis as an indicator of severe adverse maternal morbidity at a tertiary center in Southwest Nigeria. Eur J Obstet Gynecol Reprod Biol. 2018;225:205-9.

3. Heimburger O, Stenvinkel P, Barany P. The enigma of decreased creatinine generation in acute kidney injury. Nephrol Dial Transpl. 2012;27:3973-4.

4. Cheung KL, Lafayette RA. Renal physiology of pregnancy. Adv Chronic Kidney Dis. 2013;20:209-14.
Won Ho Kim

wonhokim.ane@gmail.com

1 Department of Anesthesiology and Pain Medicine, Seoul National University Hospital, 101 Daehak-ro, Jongno-gu, Seoul 03080, Republic of Korea 